\title{
Latest highlights from the ATLAS experiment
}

\author{
Pippa Wells*i \\ CERN \\ E-mail: pippa.wells@cern.ch
}

The ATLAS experiment is a general purpose detector at the CERN Large Hadron Collider. Thanks to the fantastic performance of the LHC machine and the ATLAS detector, large samples of proton-proton collisions at centre-of-mass energies of 7 and $8 \mathrm{TeV}$ have been analysed. The Standard Model of particle physics has been reestablished at these high energies, and stands firm. Measurements of soft and hard QCD processes, electroweak (di)boson production and top quark production and properties are in excellent agreement with the predictions. A new boson has been observed, with a mass of about $126 \mathrm{GeV}$ and with properties so far consistent with the Standard Model Higgs boson. However, there are still no hints of physics beyond the Standard Model from direct searches - these remain a goal of the future LHC programme.

Proceedings of the Corfu Summer Institute 2012

September 8-27, 2012

Corfu, Greece

* Speaker.

†n behalf of the ATLAS Collaboration 


\section{The ATLAS experiment}

The ATLAS experiment [1] is a multipurpose particle physics detector with forward-backward symmetric cylindrical geometry, see Fig. 1. The inner tracking detector consists of a silicon pixel detector, a silicon microstrip detector, and a straw-tube transition radiation tracker. The inner detector is surrounded by a thin superconducting solenoid which provides a $2 \mathrm{~T}$ magnetic field, and by high-granularity liquid-argon (LAr) sampling electromagnetic calorimetry. The electromagnetic calorimeter is divided into a central barrel (pseudorapidity ${ }^{1}|\eta|<1.475$ ) and end-cap regions on either end of the detector $(1.375<|\eta|<2.5$ for the outer wheel and $2.5<|\eta|<3.2$ for the inner wheel). In the region matched to the inner detector $(|\eta|<2.5)$, it is radially segmented into three layers. The first layer has a fine segmentation in $\eta$ to facilitate $e / \gamma$ separation from $\pi^{0}$ and to improve the resolution of the shower position and direction measurements. In the region $|\eta|<1.8$, the electromagnetic calorimeter is preceded by a presampler detector to correct for upstream energy losses. An iron-scintillator/tile calorimeter gives hadronic coverage in the central rapidity range $(|\eta|<1.7)$, while a LAr hadronic end-cap calorimeter provides coverage over $1.5<|\eta|<3.2$. The forward regions $(3.2<|\eta|<4.9)$ are instrumented with LAr calorimeters for both electromagnetic and hadronic measurements. The muon spectrometer surrounds the calorimeters and consists of three large air-core superconducting magnets providing a toroidal field, each with eight coils, a system of precision tracking chambers, and fast detectors for triggering. The combination

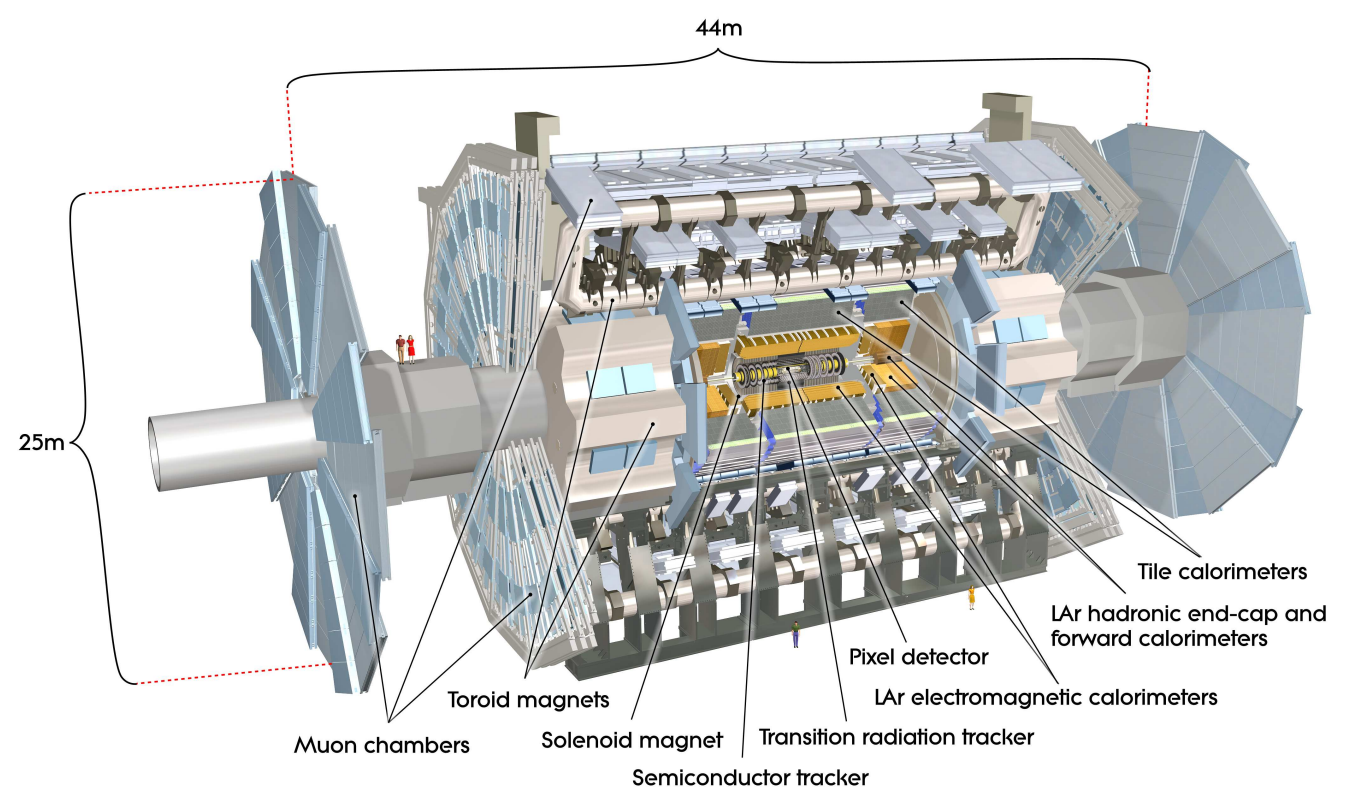

Figure 1: A cut-away view of the ATLAS detector.

\footnotetext{
${ }^{1}$ ATLAS uses a right-handed coordinate system with its origin at the nominal interaction point in the centre of the detector, and the $z$-axis along the beam line. The $x$-axis points from the origin to the centre of the LHC ring, and the $y$ axis points upwards. Cylindrical coordinates $(r, \phi)$ are used in the transverse plane, $\phi$ being the azimuthal angle around the beam line. Observables labelled "transverse" are projected into the $x-y$ plane. The pseudorapidity is defined in terms of the polar angle $\theta$ as $\eta=-\ln \tan (\theta / 2)$.
} 


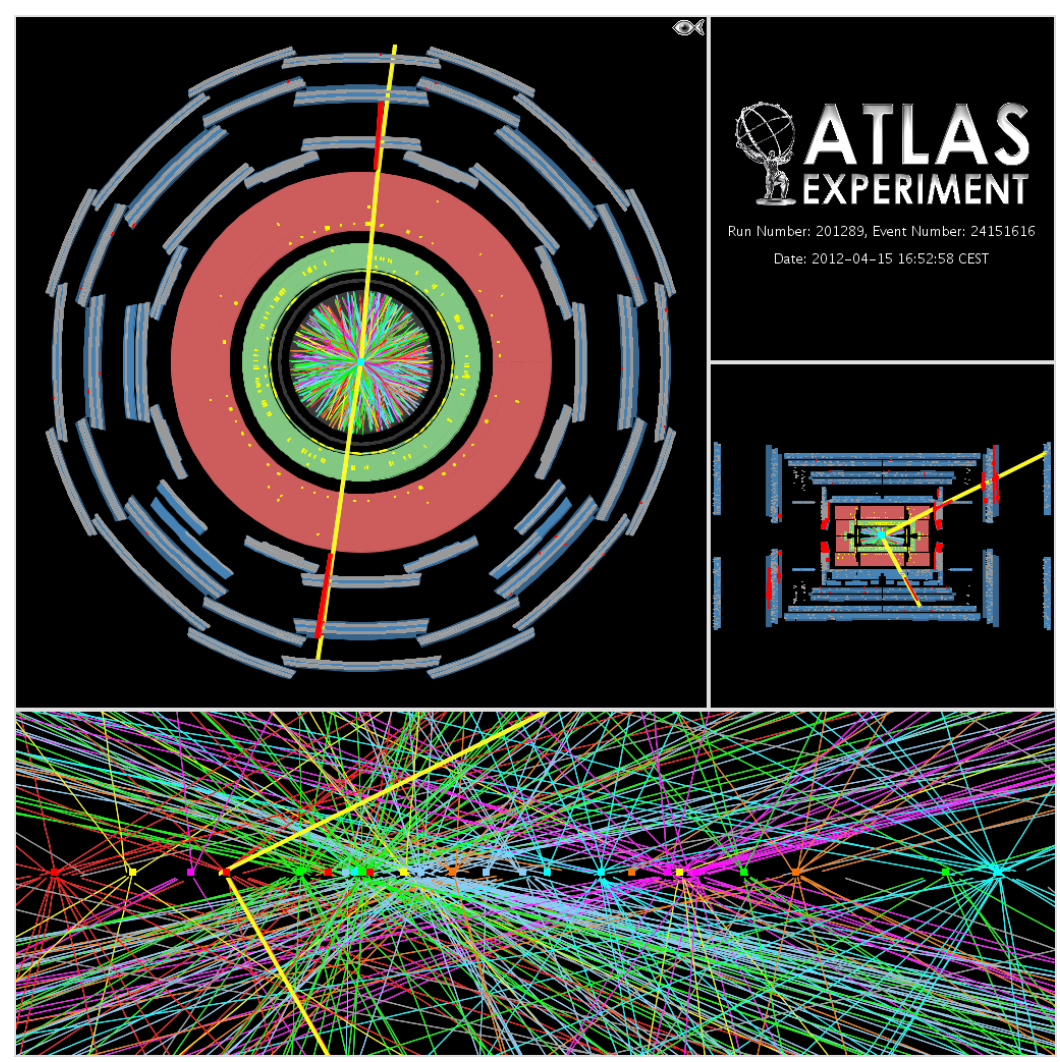

Figure 2: A $Z \rightarrow \mu^{+} \mu^{-}$event with 25 reconstructed vertices, from 15 April 2012.

of all these systems provides charged particle measurements together with efficient and precise lepton and photon measurements in the pseudorapidity range $|\eta|<2.5$. Jets and missing transverse energy, $E_{\mathrm{T}}^{\mathrm{miss}}$, are reconstructed using energy deposits over the full coverage of the calorimeters, $|\eta|<4$.9.

The Large Hadron Collider (LHC) delivered $5 \mathrm{fb}^{-1}$ of proton-proton collisions at $7 \mathrm{TeV}$ centreof-mass energy in 2011, and by the time of the Corfu Summer Institute in 2012, a further $14 \mathrm{fb}^{-1}$ at $8 \mathrm{TeV}$. The peak luminosity gradually increased over the two years. The machine is running with a higher than design number of protons per bunch, but twice the nominal bunch spacing of $50 \mathrm{~ns}$. As a result, there are typically up to $40 \mathrm{pp}$ interactions per bunch crossing. Algorithms to reconstruct interesting events have to be adapted to take into account this pile-up. In Fig. 2, 25 primary vertices have been successfully reconstructed along the few $\mathrm{cm}$ length of the luminous region at the centre of the beam pipe.

The experiment records data 24 hours a day, 7 days a week, and large teams of on-call experts are available to support the shift crews, resulting in a data taking efficiency of $94 \%$. More than $90 \%$ of the recorded data passes the quality criteria to be used for physics analysis, and a large fraction of the detector channels are operational $(\approx 99 \%)$. Thousands of jobs run on the Worldwide LHC Computing Grid, in a first pass to calibrate the detectors and then to fully process the data. This huge effort, occupying hundreds of physicists, engineers and technicians is behind every analysis result. 


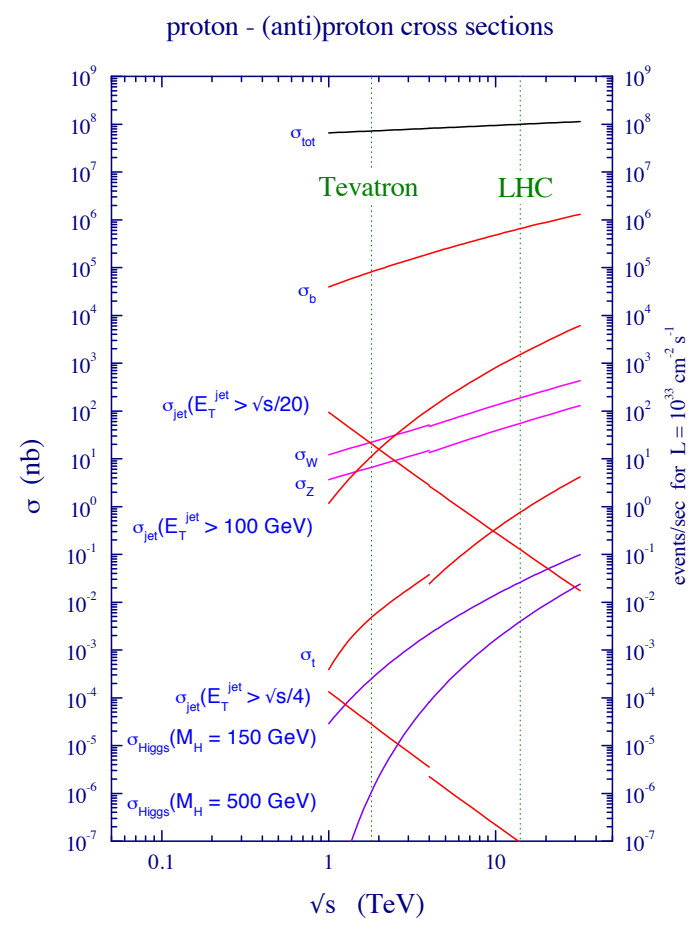

Figure 3: Production cross sections in proton-antiproton (for Tevatron) and proton-proton (for LHC) collisions as a function of centre-of-mass energy.

The total cross section is orders of magnitude larger than that of more interesting processes producing for example hard jets, $W$ and $Z$ bosons, $t \bar{t}$ pairs or even Higgs bosons, as shown in Fig. 3 A multi-level trigger system is used to decide in real time which events to record, reducing the bunch crossing rate of $40 \mathrm{MHz}$ to about 400 events per second. The trigger menus are complex, and set the minimum thresholds for objects used in the analysis.

\section{Standard Model measurements}

To model a proton-proton interaction at the LHC, parton density functions, $F\left(x, Q^{2}\right)$, describe the initial proton constituents, i.e. valence quarks, sea quarks and anti-quarks, and gluons. The hard scatter is calculated at NLO, NNLO or higher. The proton remnants not involved in the hard scatter create the underlying event. Final state partons fragment into hadrons, whose distribution can be described by fragmentation functions $D(z)$, defining the fraction of the parton momentum taken by the hadron. The final state is simulated by parton shower and hadronisation models. Theoretical calculations of the hard scatter can be compared with data either at the detector level from Monte Carlo simulations, or after unfolding. There are also many studies of the phenomenology of soft QCD. 

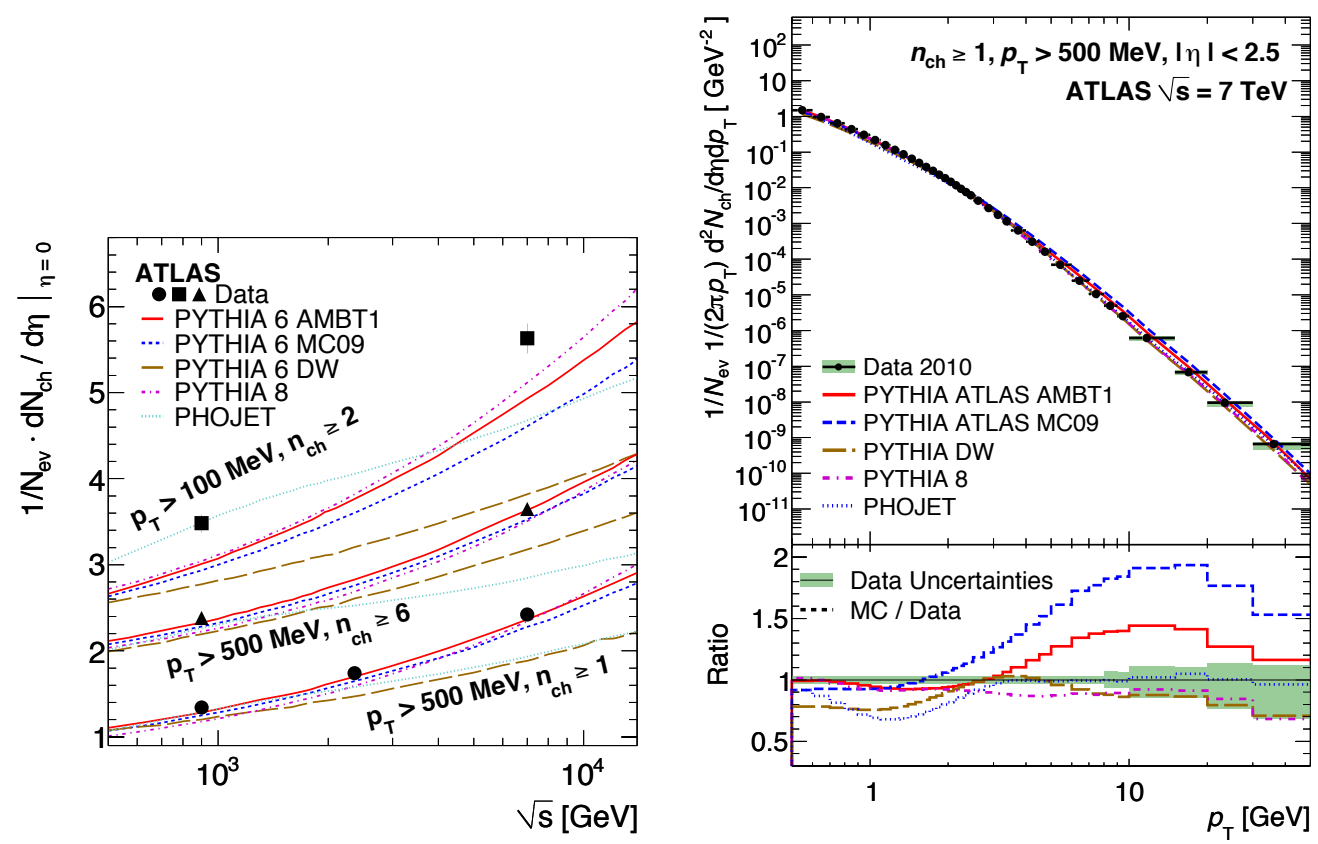

Figure 4: The charged particle density for differing $p_{\mathrm{T}}$ thresholds, as a function of centre-of-mass energy (left). The $p_{\mathrm{T}}$ distribution of charged particles in $7 \mathrm{TeV}$ collisions (right).
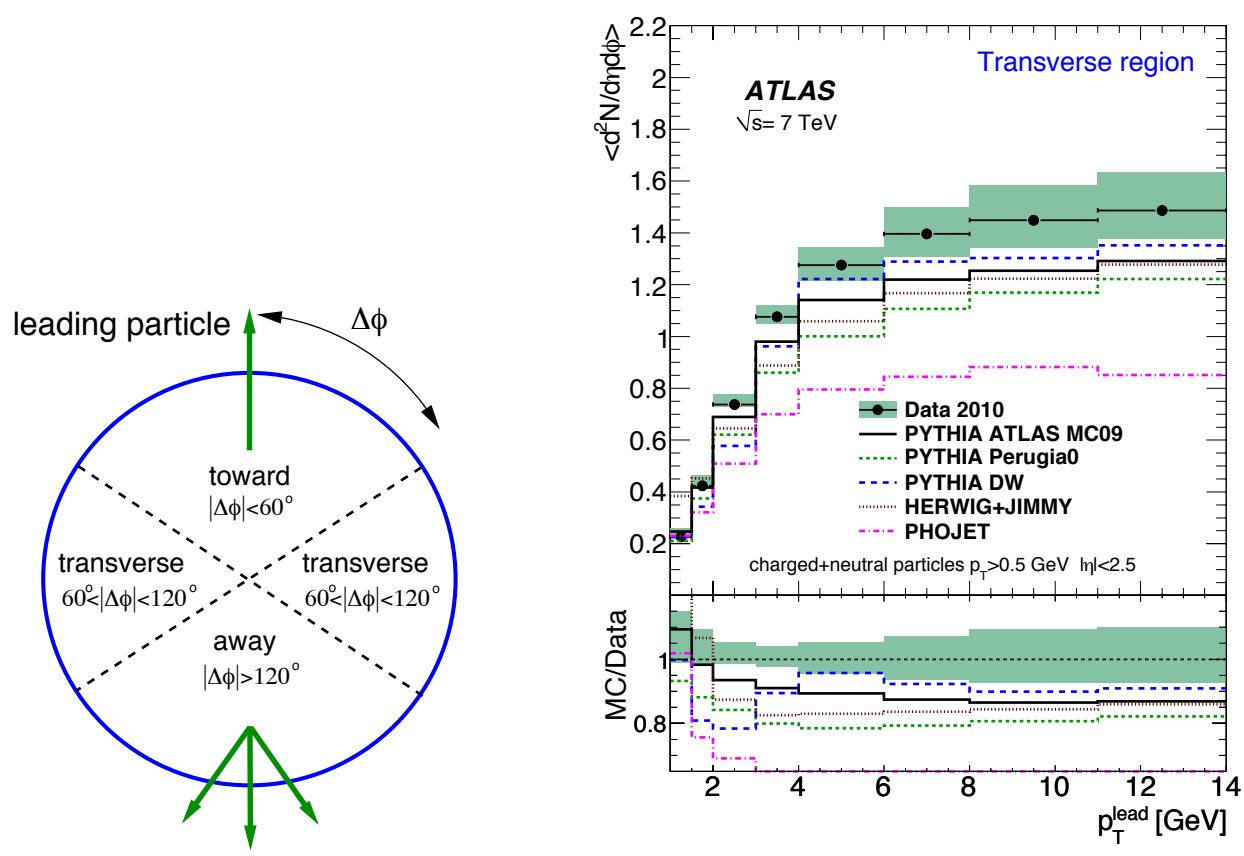

Figure 5: The definition of the transverse region in order to measure the underlying event (left), and the average number of stable particles per event per unit interval in $\eta-\phi$ in the transverse region, as a function of the $p_{\mathrm{T}}$ of the leading particle. 
The first collisions at the LHC were at $900 \mathrm{GeV}$ centre-of-mass energy in 2009, followed by $7 \mathrm{TeV}$ in 2010. The first published measurements [2] were of charged particle production in minimum bias events, which already required a solid understanding of the detector performance and details of the detector geometry. Detailed measurements of the properties of minimum bias events have been made with low integrated luminosity and low pile-up, see for example Fig. 4 [3]. These measurements can then be used to improve the Monte Carlo description of the data.

The properties of the underlying event can be probed by looking in the region away from the direction of the leading track in the event [4]. An example of the quality of the description of the underlying event is shown in Fig. 5.

Jets are identified using the anti- $\mathrm{k}_{t}$ algorithm, controlled by a distance parameter $\mathrm{R}$ in the $(\phi, y)$ plane. The pair of particles with minimum $d_{i j}$ are merged, unless $d_{i \text {, beam }}$ is smallest, in which case the $i$ th object becomes a jet:

$$
d_{i j}=\min \left(\frac{1}{k_{t i}^{2}}, \frac{1}{k_{t j}^{2}}\right) \frac{\Delta \phi^{2}+\Delta y^{2}}{R^{2}} ; d_{i, \text { beam }}=\frac{1}{k_{t i}^{2}} .
$$

Jets are reconstructed from three-dimensional clusters of energy deposits in the calorimeter, initially at the electromagnetic scale, which is calibrated using $Z \rightarrow e e$ events. Corrections are made for the contributions from pile-up events and instrumental defects. The energy is then adjusted to the hadronic scale using $\eta$ and energy dependent factors from simulation, but verified by in-situ measurements in data. The jet energy scale uncertainty is between 2.5 and $4.6 \%$ for the central rapidity region (in 2010). Inclusive jet production has been measured with individual jet $p_{\mathrm{T}}$ up to more than $1 \mathrm{TeV}$, and rapidity in the range $|y|<4.4$, for distance parameters $R=0.4$ and 0.6. These are compared to NLO calculations with non-perturbative corrections, and very good agreement is seen within the systematic uncertainties, as shown in Fig. 6
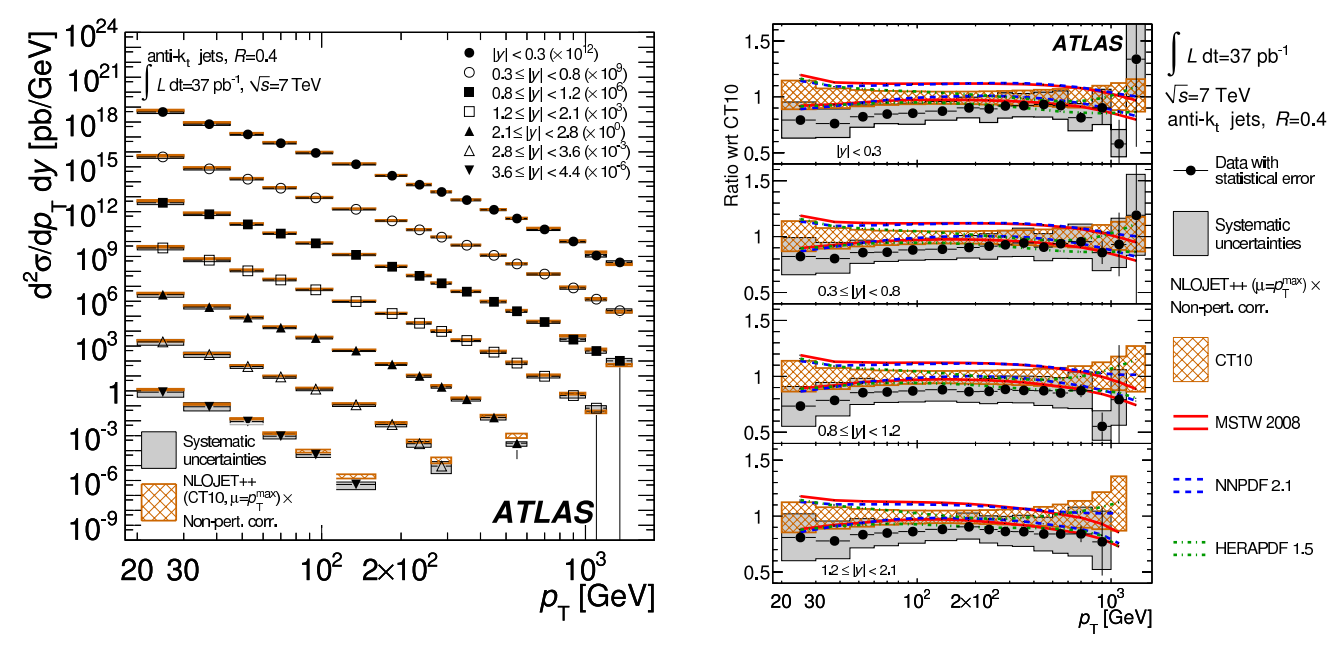

Figure 6: (left) Inclusive jet double-differential cross section as a function of jet $p_{\mathrm{T}}$ in different regions of $|y|$ for jets with $R=0.4$. The cross sections are multiplied by the factors indicated in the legend. (right) Ratios of inclusive jet double-differential cross section to the theoretical prediction. 

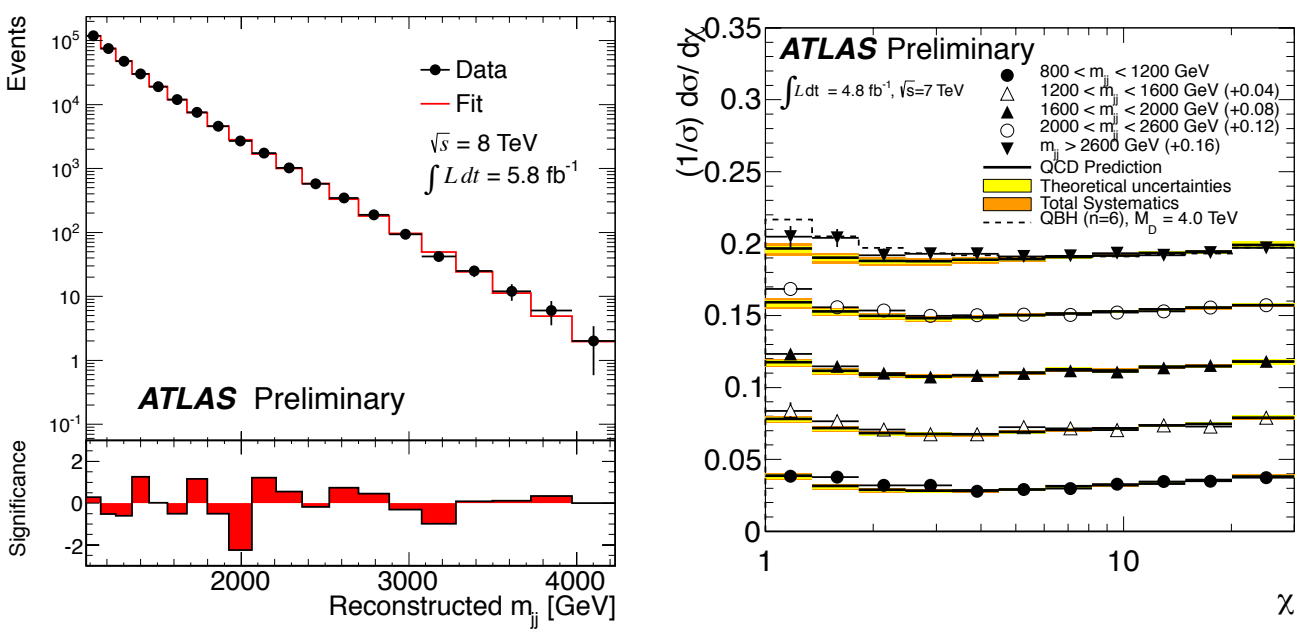

Figure 7: (left) The reconstructed dijet mass distribution with statistical uncertainties (filled points with error bars) fitted with a smooth functional form (solid line). The bin-by-bin significance of the data-fit difference is shown in the lower panel, using positive values for excesses and negative values for deficits. (right) The 11-bin angular distributions for all dijet mass bins. The QCD predictions are shown with theoretical and total systematic uncertainties (bands), as well as the data with statistical uncertainties. The dashed line is the prediction for an example quantum black hole signal in the highest mass bin. The distributions have been offset by the amount shown in the legend.

The dijet mass spectrum extends to about $4 \mathrm{TeV}$, and has been measured as a function of rapidity. The rate and distribution of multijet events has also been studied [6]. The dijet mass spectrum is smoothly falling, as seen in Fig. 7 (left). A search for significant bumps has been performed using $8 \mathrm{TeV}$ data. An excited quark with mass less than $3.66 \mathrm{TeV}$ is excluded at $95 \%$ confidence level (CL) [7]. Including information from angular variables, more physics models beyond the Standard Model (SM) can be tested. Contact interactions are excluded up to $7.8 \mathrm{TeV}$. In Fig. 7 (right), the modification to the angular variable $\chi=\exp \left(\left|y_{1}-y_{2}\right|\right)$ due to a hypothetical quantum black hole is illustrated.

Electron identification in ATLAS starts with energy clusters in the calorimeter, which are required to satisfy shower shape and isolation criteria. There must be an inner detector track matched to the cluster, with the ratio of cluster energy to track momentum close to unity. There must also be a large fraction of high threshold transition radiation tracker hits, consistent with transition radiation. The electron reconstruction is improved by accounting for Bremsstrahlung photons. During 2011, the algorithms were refined to be robust against pile-up.

Muons are identified by matching track segments in the inner detector and muon spectrometer. The muon spectrometer information also improves the momentum reslution for high $p_{\mathrm{T}}$ muons. Muons with low momentum, or in regions of the detector with no muon chambers, can be recovered by matching an inner detector track with minimal energy loss in the calorimeter.

Dedicated algorithms identify hadronic decays of tau-leptons, which produce very narrow jets with low track multiplicity from the 1- and 3-prong decays. The electron or muon from leptonic tau decays can also be detected. 
Even in a small data set, the dilepton mass spectrum shows the well known resonances, up to and including the $Z$ boson, which can be used to verify the energy or momentum calibration. An example is shown in Fig. 8 for muon pairs in the 2010 data set. The upper end of the spectrum is used to search for new states such as a $Z^{\prime}$ boson.

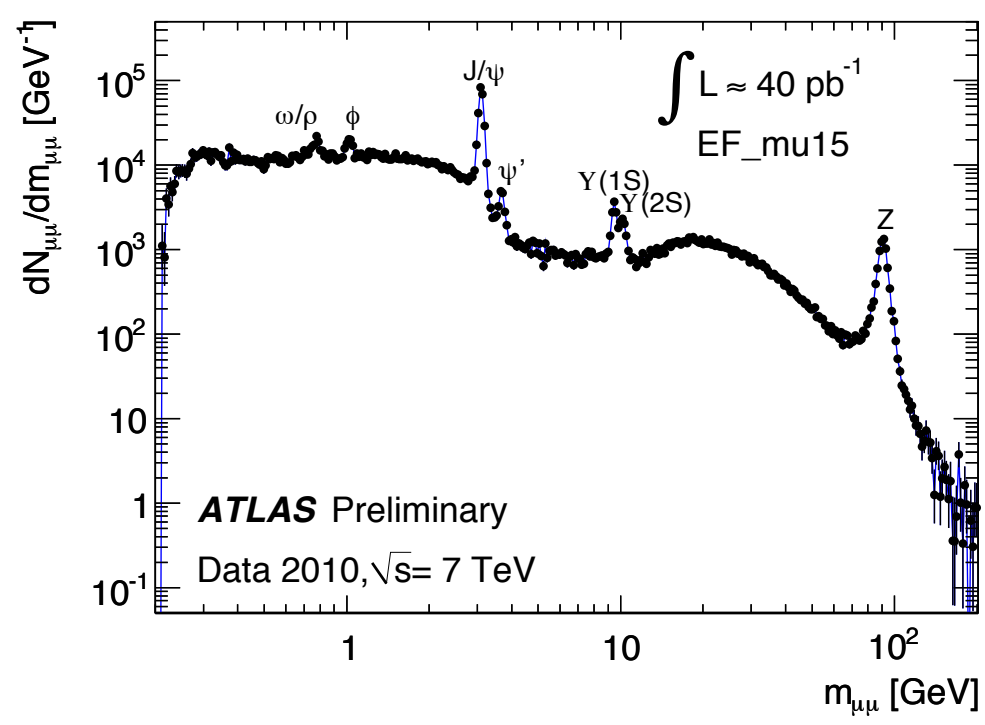

Figure 8: Dimuon invariant mass spectrum for the full 2010 data set.

Undetectable particles, such as neutrinos, are inferred by measuring the transverse energy balance of the event. The missing transverse energy, $E_{\mathrm{T}}^{\mathrm{miss}}$, is the magnitude of the vector some of the transverse momentum vectors of particles in the event. The ATLAS $E_{\mathrm{T}}^{\text {miss }}$ resolution is $0.5 \cdot \Sigma E_{\mathrm{T}}$, and this has been verified up to $\Sigma E_{\mathrm{T}}$ of $14 \mathrm{TeV}$ using lead-lead collision data. The $E_{\mathrm{T}}^{\text {miss }}$ resolution is improved by using the correct energy scale for identified objects (leptons, jets, etc.). Tracks are used to make the algorithms more robust against pileup, by working out which energy deposites are associated with the primary vertex of the hard ineraction.

$W$ and $Z$ boson leptonic decays have been measured [9]. The $W$ decay to a charged lepton and neutrino is reconstructed from the measured lepton and $E_{\mathrm{T}}^{\mathrm{miss}}$. A transverse mass variable is calculated, since the longitudinal component of the neutrino momentum is unknown. The $Z$ boson mass can be measured directly from a pair of same flavour, oppositely charged leptons. Decays with tau-leptons in the final state have also been measured. In contrast, hadronic $W$ and $Z$ decays are swamped by background from QCD processes. Examples of the clean signal and measured cross sections are shown in Fig. 9.

The transverse momentum spectrum of $W$ and $Z$ bosons, and production in association with jets have also been studied $[10,11]$ and are reasonably well described. In addition, the data samples are large enough to measure diboson production, including $W \gamma, Z \gamma, W W, W Z$ and $Z Z$ [12]. The leptonic final states $W W \rightarrow \ell v \ell v, W Z \rightarrow \ell v \ell \ell$ and $Z Z \rightarrow \ell \ell \ell \ell$ have been observed, while it is much more difficult to distinguish final states with jets, $\ell v j j$ or $\ell \ell j j$, from jet production in association with a single boson. These measurements can constrain possible anomalous triple gauge couplings. 
The charged TGCs, $W W \gamma$ and $W W Z$, are non-zero in the Standard Model, while the neutral TGCs, $Z Z \gamma$ and $Z \gamma \gamma$ are strictly zero. No deviations from the expectation are observed, and the ATLAS constraints are already more powerful than those from the Tevatron.

Top quark pairs $t \bar{t}$ are characterised by the the decay of each top $\approx 100 \%$ of the time to $W b$, with the $W$ decay to $\ell v$ or jets, leading to combinations of leptons, $E_{\mathrm{T}}^{\mathrm{miss}}$ and/or jets from the $W$ decays, together with two $b$-jets. The $b$-jets can be tagged by the resolvable lifetime of the $b$-hadron, resulting in tracks with significant impact parameter, and possibly a reconstructed secondary vertex with characteristic mass. Soft muons in the $b$-jet from weak decays may also be identified. It was already possible to observe $t \bar{t}$ production in the 2010 data sample, and the present status of $t \bar{t}$ cross-section measurements is shown in Fig. 10, achieving a 6\% relative precision [13]. Sufficient statistics are available to make relative differential cross-section measurements [14], an example of which is also shown in Fig. 10. The most precise measurement of the top quark mass to date by ATLAS [15] is made using a template method in the lepton+jets final state: $m_{\text {top }}=174.5 \pm$ 0.6 (stat) \pm 2.3 (syst) GeV. The dominant uncertainty is the jet energy scale, in particular the $b$-jet energy scale
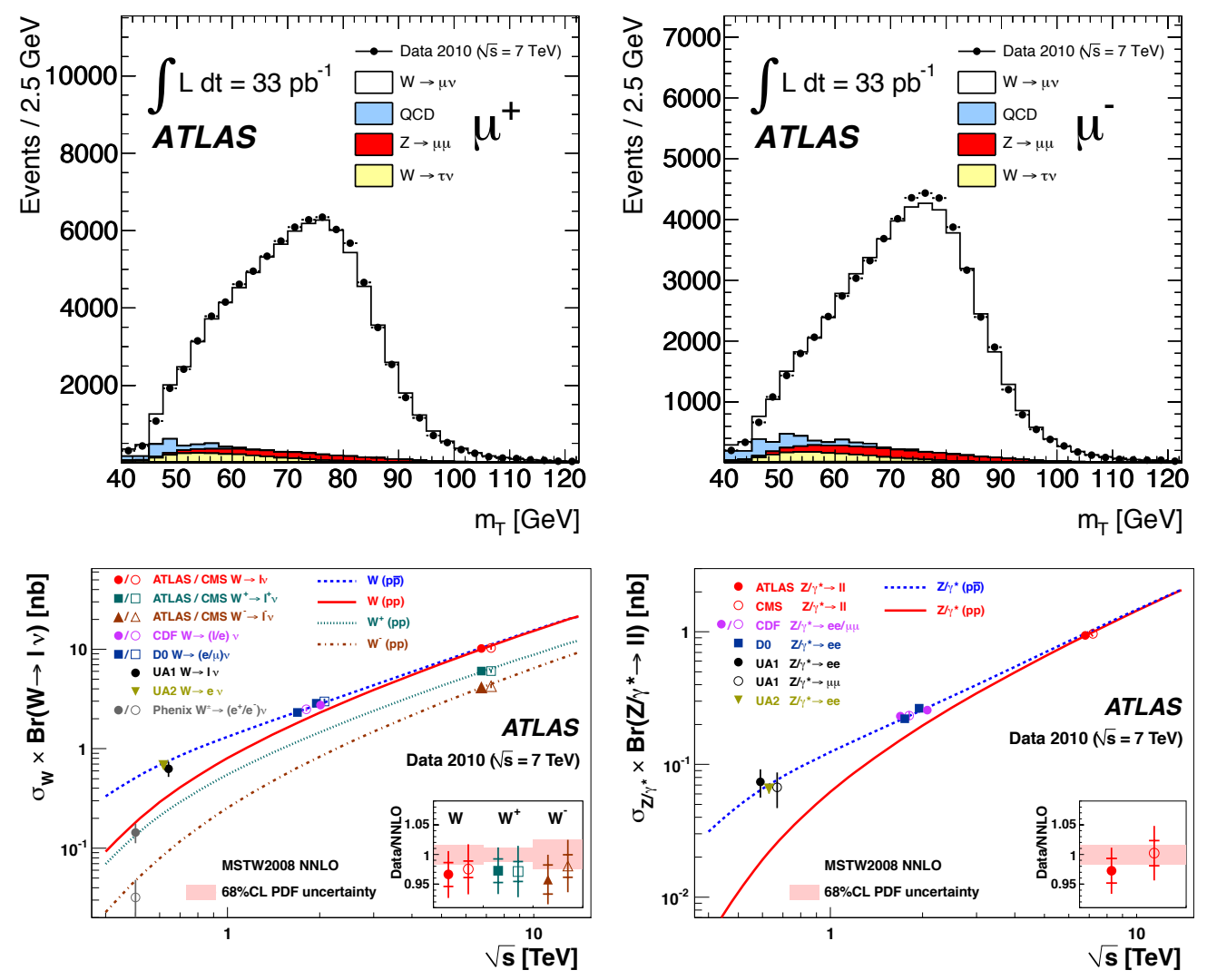

Figure 9: Transverse mass distribution of candidate $W^{+}$(top left) and $W^{-}$(top right) events. The simulation is normalised to the data. The QCD background shape is taken from simulation and normalised to the number of QCD events measured from data. The measured values of $\sigma(W) \times \mathrm{BR}(W \rightarrow \ell v)$ (bottom left) and $\sigma(Z) \times \mathrm{BR}(Z \rightarrow \ell v)$ (bottom right) in $p \bar{p}$ and $p p$ collisions as a function of centre-of-mass energy. 

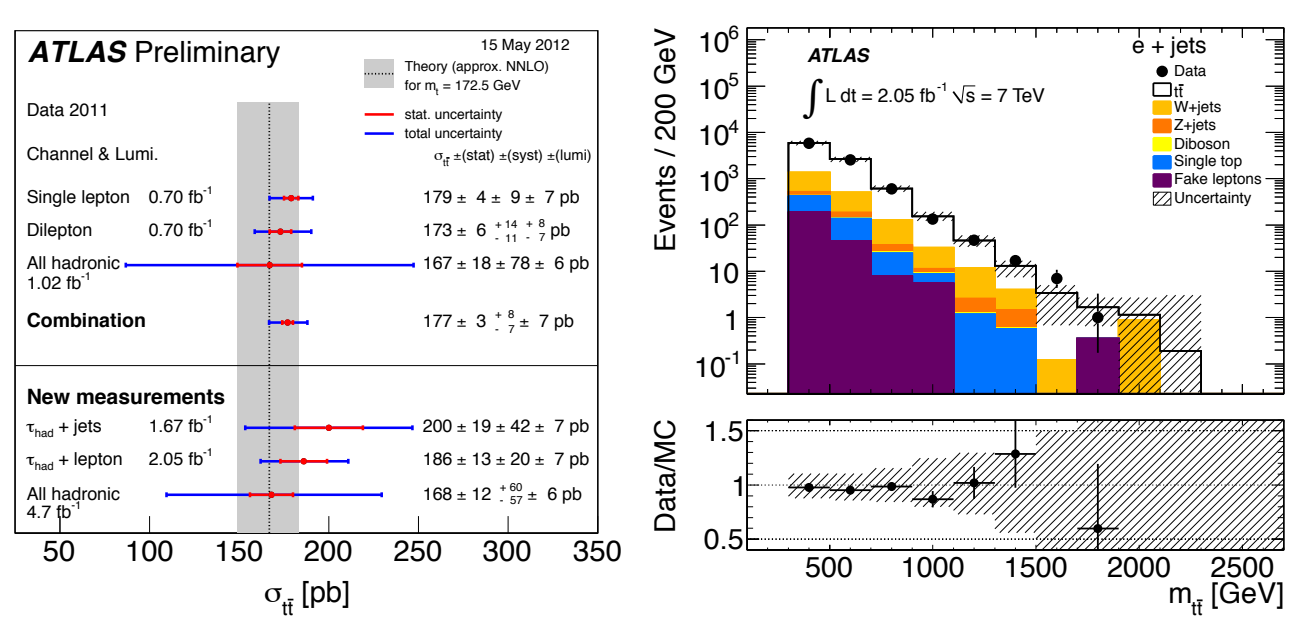

Figure 10: Measurements of the $t \bar{t}$ production cross section, and their combination (left) and an example differential measurement as a function of the $t \bar{t}$ mass (right).

The forward-backward charge asymmetry for $t \bar{t}$ events which has been of particular interest in $p \bar{p}$ events at the Tevatron becomes an asymmetry in the (pseudo)-rapidity difference in $p p$ collisions. The top quark tends to have larger $|\eta|$ or $|y|$ than the antiquark. An asymmetry is defined as:

$$
A_{C}=\frac{N(\Delta|y|>0)-N(\Delta|y|<0)}{N(\Delta|y|>0)+N(\Delta|y|<0)}
$$

where $\Delta|y|=|y(t)|-|y(\bar{t})|$. The theoretical expection is $A_{C}=0.006 \pm 0.002$, compared to the present measurement after unfolding of $A_{C}=0.019 \pm 0.028$ (stat) \pm 0.024 (syst) [16].

To complete the picture, single top production has been studied in the $t$-channel, $W t$-channel and $s$-channel modes. A summary of all the Standard Model cross-section measurements is shown in Fig. 11, with good agreement between measurement and predictcion for processes spanning several orders of magnitude. The Standard Model continues to describe measurements at the LHC, as it has to per mille precision at LEP and the Tevatron, which requires at least one Higgs boson, or something else to play its role. 


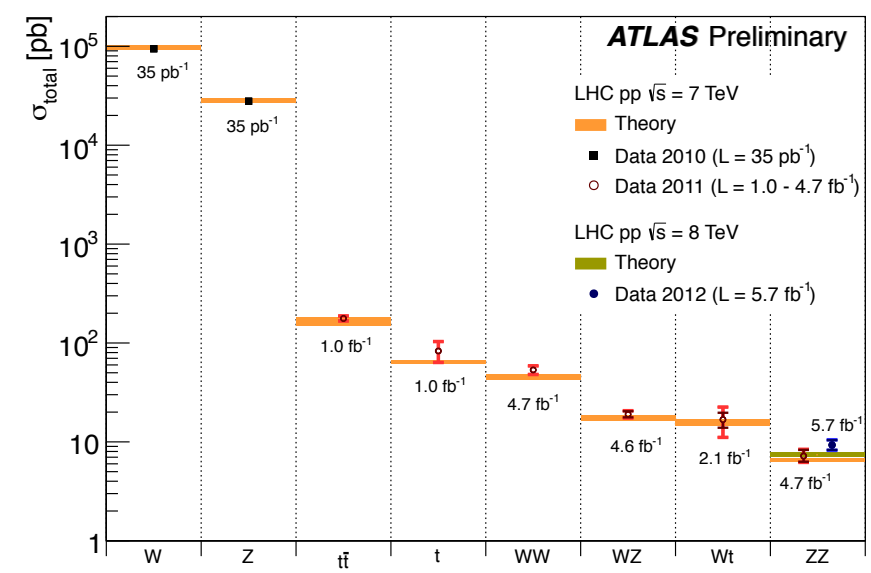

Figure 11: Summary of several Standard Model total production cross-section measurements, corrected for leptonic branching fractions, compared to the corresponding theoretical expectations. The year of data taking, centre-of-mass energy and the size of the data sample is indicated for each result.
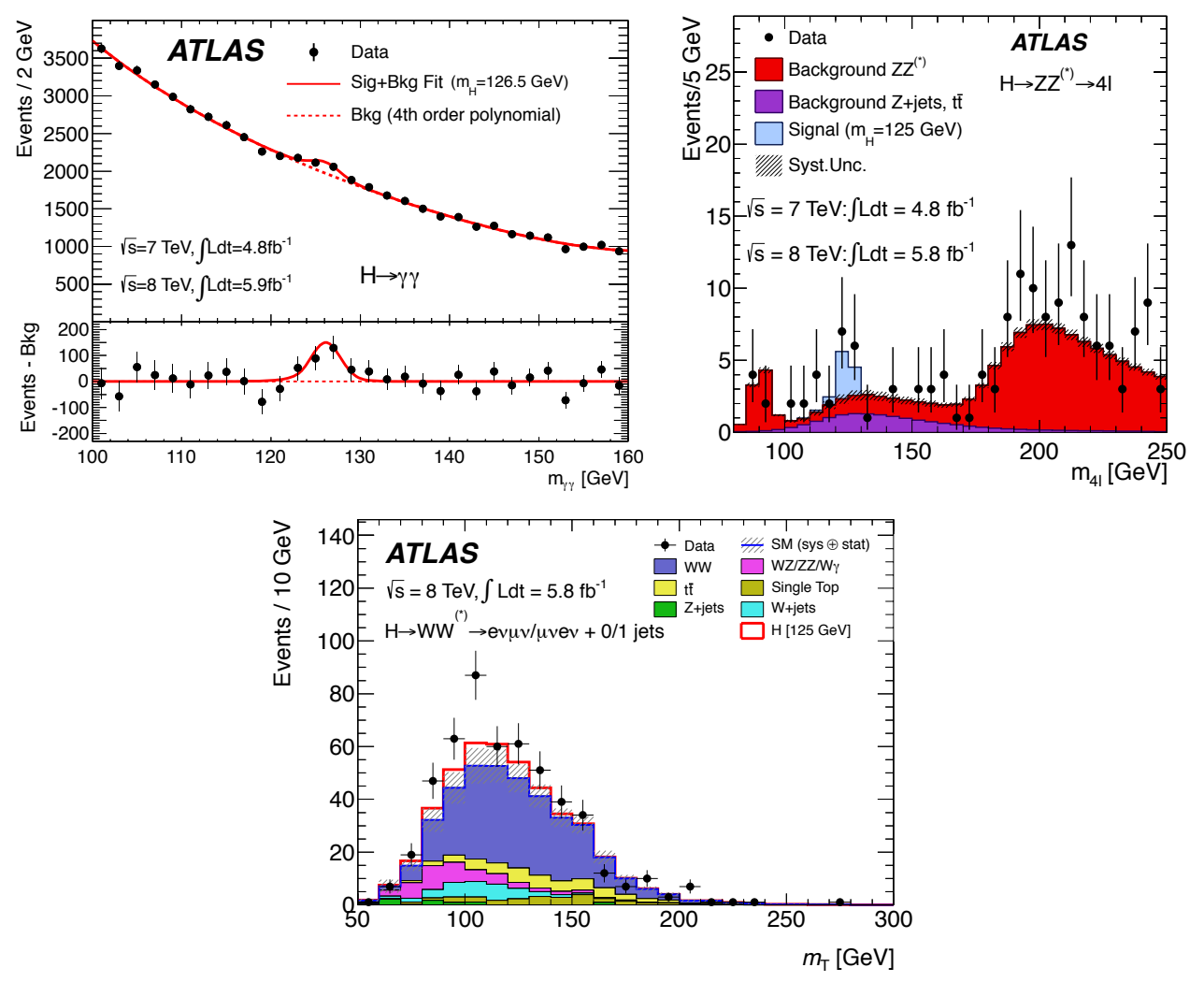

Figure 12: (top left) The mass distribution of diphoton events, with a visible excess over background at about $125 \mathrm{GeV}$. (top right) The invariant mass of the four lepton system in the search for $H \rightarrow Z Z$, with a visible excess in the same region. Decays of a single $Z$ boson form the expected peak at $90 \mathrm{GeV}$, while standard model $Z Z$ rises to a maximum around $200 \mathrm{GeV}$. (bottom) The transverse mass distribution of $W W$ events, showing a broad excess consistent with Higgs boson production with $m_{\mathrm{H}}=125 \mathrm{GeV}$. 


\section{Search for the Higgs boson and observation of a new particle}

The search for the Higgs boson is one of the main goals of the LHC programme. The choice to extend Run 1 to the end of 2012 was with the aim of observing or excluding a Higgs boson across the whole allowed mass range. Higgs production and decay has been calculated to the best available precision by a joint working group of experimentalists and theorists [17]. Higgs production is dominated by gluon fusion via a fermion loop. Vector boson fusion is typically an order of magnitude lower, but characterised by additional jets in the final state, and depending directly on the Higgs coupling to bosons. Associated production of a Higgs boson with a $W, Z$ or $t \bar{t}$ allows access to final states which are experimentally more difficult to pick out from backgrounds, such as $b \bar{b}$. The production rates must then be combined with the Higgs branching ratios to find the channels with the best signal to background. The Higgs boson tends to decay to the highest mass final state particles available. The $W W^{*}$ and $Z Z^{*}$ final states give the best sensitivity at high mass, while $H \rightarrow \gamma \gamma$ (via a loop) gives a clean signal in the low mass region, despite the small branching ratio, because of the good mass resolution.

The ATLAS Higgs boson search in 2012 [18] focussed first on the high-sensitivity channels for the lower mass range, where a hint of a signal had been observed in the 2011 data. To identify $H \rightarrow$ $\gamma \gamma$ requires rejection factors of $10^{4}$ against $\gamma$-jet events, and $10^{7}$ against jet-jet events. The main background is from jets with a leading $\pi^{0}$. The fine segmentation of the ATLAS electromagnetic calorimeter is important in rejecting these, and in addition gives pointing information to associate the photon with the primary vertex. The rate of non-photon backgrounds can be established by fitting the data directly. The diphoton mass resolution depends on the photon energy resolution and the resolution on the opening angle between them. Tracking information from converted photons can be combined with pointing information from the calorimeter. The typical mass resolution is 1.5 to $2.0 \mathrm{GeV}$, depending on the region of the detector and whether the photon has converted. The data are further subdivided according to the $p_{\mathrm{T}}$ of the diphoton system relative to its thrust, and the presence of additional jets, and the analysis is performed in 10 different channels.

The inclusive diphoton mass spectrum is shown in Fig. 12. The distribution is about $70 \%$ pure in genuine $\gamma \gamma$ events, and both the reducible and irreducible backgrounds to the Higgs boson signal are smoothly falling. There is a visible excess of events around $125 \mathrm{GeV}$ [18].

\begin{tabular}{|l|l|l|l|}
\hline $\begin{array}{l}\text { Integrated luminosity } \\
\text { and mass range }\end{array}$ & $\begin{array}{l}7 \mathrm{TeV} \\
{\left[\mathrm{fb}^{-1}\right]}\end{array}$ & $\begin{array}{l}8 \mathrm{TeV} \\
{\left[\mathrm{fb}^{-1}\right]}\end{array}$ & $m_{H}[\mathrm{GeV}]$ \\
\hline$H \rightarrow \gamma \gamma$ & 4.8 & 5.9 & $110-150$ \\
$W, Z+H \rightarrow b b$ & 4.7 & - & $110-130$ \\
$H \rightarrow \tau \tau$ & 4.7 & - & $110-150$ \\
$H \rightarrow W W \rightarrow \ell v \ell v$ & 4.7 & $5.8(e \mu$ only $)$ & $110-600(110-200)$ \\
$H \rightarrow W W \rightarrow \ell v q q$ & 4.7 & - & $300-600$ \\
$H \rightarrow Z Z \rightarrow \ell \ell \ell \ell$ & 4.8 & 5.8 & $110-600$ \\
$H \rightarrow Z Z \rightarrow \ell \ell q q$ & 4.7 & - & $200-600$ \\
$H \rightarrow Z Z \rightarrow \ell \ell v v$ & 4.7 & - & $200-600$ \\
\hline
\end{tabular}

Table 1: The channels included in the combined Higgs search results from 7 and $8 \mathrm{TeV}$ data 
The decay $H \rightarrow Z Z \rightarrow \ell \ell \ell \ell$ is considered the gold-standard search channel, with a very clean signature of two pairs of isolated, same-flavour, opposite-charge leptons. The four-lepton system also has very good mass resolution. In this case the expected background, also shown in Fig. 12, is not simply a smoothly falling spectrum, but it is very well described by Monte Carlo simulation. An excess of events is visible at about the same mass as the excess in the diphoton sample.

The third channel offering high sensitivity is $H \rightarrow W W \rightarrow \ell v \ell v$, but the two neutrinos are only inferred from the $E_{\mathrm{T}}^{\text {miss }}$ in the event. A transverse mass variable can be reconstructed, which has a broad predicted contribution from Higgs boson decays. The analysis is restricted to $e v \mu v$ final states at this stage, to suppress the $Z$ decay background. A small opening angle is required between the leptons to improve the signal-to-background. More events are observed in data than expected from other processes, as shown in Fig. 12.

The three new analysis using 2012 data are combined with the results in the full set of final states from $7 \mathrm{TeV}$ running in 2011. The channels, integrated luminosities and the Higgs mass range for which they are used are listed in Table 1. Each of these channels is further divided into numerous sub-channels for optimal sensitivity. All the searches are combined together. The signal
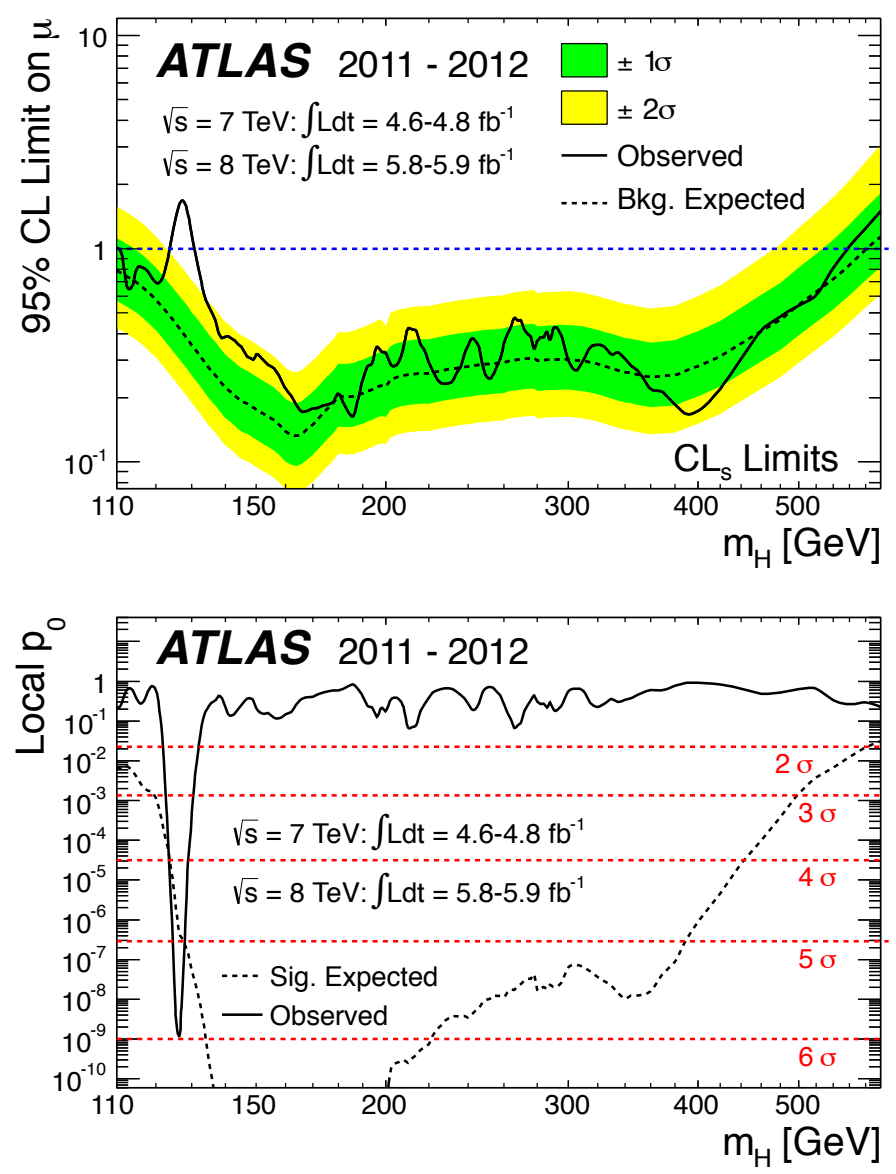

Figure 13: Excluded Higgs boson cross section expressed as the ratio $\mu$ to the Standard Model cross section (top), and the consistency with a background fluctuation (bottom). Further explanation is given in the text. 
cross section excluded at $95 \%$ confidence level is shown in Fig. 13 as a function of the Higgs boson mass, with the signal strengh expressed as the ratio $\mu$ to the Standard Model prediction. A Standard Model Higgs boson is excluded at $95 \%$ confidence level in the range $111-122 \mathrm{GeV}$ (i.e. meeting the range below $114 \mathrm{GeV}$ already excluded by the LEP experiments), and from $131-559 \mathrm{GeV}$. The exclusion is greater than $99 \%$ over a large part of this range.

The probability that the observation is consistent with background processes is also shown in Fig. 13. The observed local p-value for each mass hypothesis is shown as the solid line. The dotted line shows the expected p-value in the case of Standard Model Higgs production at each Higgs mass. The minimum p-value is $1.7 \times 10^{-9}$, corresponding to a significance of 5.9 standard deviations [18].

The 68 and 95\% confidence intervals from a fit where both the signal strength and the mass are free are shown in Fig. 14. Combining the precise mass measurements from the diphoton and $Z Z \rightarrow 4 \ell$ channels, without constraining the signal strength, the best fit mass is $126.0 \pm 0.4$ (stat) \pm 0.4 (syst) $\mathrm{GeV}$. The best fit signal strength in each channel, this time constraining the Higgs boson mass to be $126.0 \mathrm{GeV}$, is shown in Fig 14 . The overall combined signal strength is $\mu=1.4 \pm 0.3$, consistent with the Standard Model rate.
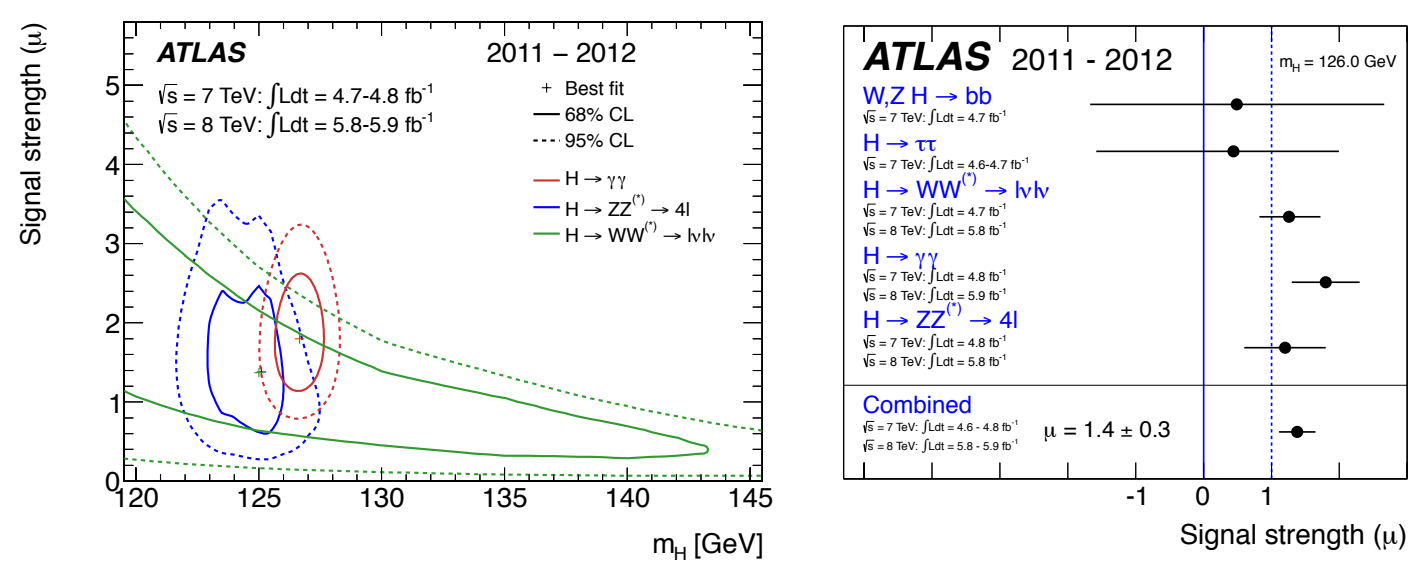

Figure 14: Confidence intervals in the $\mu$ vs mass plane for the diphoton, $Z Z$ and $W W$ channels (left), and the measured signal strength parameters in all channel, scaled to the Standard Model prediction for a Higgs boson mass of $126.0 \mathrm{GeV}$.

Searches for non-Standard Higgs bosons also continue. The Standard Model with a single Higgs boson is the minimal solution, but two Higgs-doublet models are also consistent, as for example the type-II model needed for supersymmetry. These require three neutral bosons, the light $h$ (which can have properties very similar to the Standard Model Higgs boson), a heavy $H$ which is also CP even, and a CP odd $A$. There are also two charged Higgs bosons, $H^{+}$and $H^{-}$. No hints of additional Higgs bosons have been found so far. Further measurements of the properties of the newly discovered boson will also be made to see if it remains consistent with the minimal Standard Model prediction. 


\section{Physics beyond the Standard Model}

Although the Standard Model has been phenomenally successful in predicting how particles interact, it must be extended by a more complete theory at high energies. This should extend the theory to include gravity, and address the issues of the as-yet unexplained dark matter and dark energy needed to explain the cosmos at many scales, from galaxy rotation curves to large scale structures. The theory does not explain the matter to anti-matter imbalance in the universe. In addition, it has unsatisfactory features of hierarchy, lack of naturalness and the need for fine tuning.

Supersymmetry (SUSY) is an attractive extension of the Standard Model, where all the standard particles have supersymmetric partners with half-unit different spin. No SUSY particles have been observed, so this must be a broken symmetry where the partners have higher mass. It is often assumed that there is a new conserved quantum number, $R$, with $R=+1$ for standard particles and $R=-1$ for SUSY partners, which preserves baryon and lepton number conservation. If $R$ is conserved, SUSY particles must be produced in pairs, and if the lightest SUSY partner (LSP) is charge and colour neutral it becomes a natural dark matter candidate.

The minimal supersymmetric standard model, MSSM, requires two Higgs doublets and all the superpartners, leading to 124 independent free parameters. The constrained minimal extension of the Standard Model (CMSSM) has only five parameters at the high energy Grand Unification scale: a universal scalar mass $m_{0}$; a universal gaugino mass $m_{1 / 2}$; a universal trilinear soft SUSY breaking parameter $A_{0}$; the ratio of the vacuum expectation values of two Higgs doublets, $\tan \beta$; and the sign of the Higgs mixing parameter, $\operatorname{sign}(\mu)$. The CMSSM may be convenient for interpreting the results of searches for SUSY particles, but it is more generally useful to provide results in the form of simplified models which can then be interpreted in other scenarios. In this case cross-section limits or excluded mass regions are provided as a function of for example a squark and gluino mass. In general the SUSY phenomenology depends on mass splittings between squark or gluino, the LSP and standard particles

SUSY production cross sections have a logarithmic dependence on the particle mass, which leads to a gain in sensitivity with increasing luminosity. At the sensitivity limit of around a TeV, there is a further effective gain in parton luminosity of around a factor 2 to 5 by increasing the proton-proton centre-of-mass energy from 7 to $8 \mathrm{TeV}$.

There is a very rich phenomenology of SUSY final states, spanning short or long decay chains, often containing jets and large $E_{\mathrm{T}}^{\text {miss }}$, but also leptons, $b$-jets, photons or even long-lived hadrons. The analyses are divided according to the observable topologies and then interpreted in specific SUSY models.

A few new SUSY search results using $8 \mathrm{TeV}$ are already available, including a search with jets and $E_{\mathrm{T}}^{\text {miss }}$ in the final state [19]. The analysis is optimised for discovery in a simplified model with a low mass LSP, observable (first and second generation) squarks and gluinos, and with all other SUSY particles pushed to high mass. This results in short decay chains, $\tilde{q} \rightarrow q \tilde{\chi}^{0}$ or $\tilde{g} \rightarrow$ $\tilde{q} q \rightarrow q q \tilde{\chi}^{0}$. Events are selected with a jet or $E_{\mathrm{T}}^{\text {miss }}$ trigger, and a veto on events with leptons. Tight, medium and loose signal regions are defined according to the number of jets and the value of $m_{\mathrm{eff}}=\sum_{i}\left|\vec{p}_{\mathrm{T}}{ }^{\text {jet }, i}\right|+E_{\mathrm{T}}^{\text {miss }}$, as specified in Table 2. Four control regions are defined for each signal region, to control the rates of $Z \rightarrow v v+$ jets, $W+$ jets, $t \bar{t}$ and multijet background. Transfer functions are used to infer the background in the signal region, and combined likelihood fits to 


\begin{tabular}{|l|c|c|c|c|c|}
\hline Requirement & 2-jets & 3-jets & 4-jets & 5-jets & 6-jets \\
\hline$E_{T}^{\text {miss }}[\mathrm{GeV}]>$ & \multicolumn{5}{|l}{} \\
$p_{\mathrm{T}}$ jet $1[\mathrm{GeV}]>$ & \multicolumn{5}{|l|}{160} \\
$p_{\mathrm{T}}$ jet $2[\mathrm{GeV}]>$ & \multicolumn{5}{|c|}{130} \\
\hline$p_{\mathrm{T}}$ jet $3[\mathrm{GeV}]>$ & - & 60 & 60 & 60 & 60 \\
$p_{\mathrm{T}}$ jet $4[\mathrm{GeV}]>$ & - & - & 60 & 60 & 60 \\
$p_{\mathrm{T}}$ jet $5[\mathrm{GeV}]>$ & - & - & - & 60 & 60 \\
$p_{\mathrm{T}}$ jet $6[\mathrm{GeV}]>$ & - & - & - & - & 60 \\
\hline$\Delta \phi\left(j, E_{T}^{\text {miss }}\right)[\mathrm{rad}]$ & \multicolumn{2}{|c|}{$0.4(\mathrm{j} \leq 3)$} & $0.4(\mathrm{j} \leq 3), 0.2\left(p_{\mathrm{T}}>40 \mathrm{GeV}\right.$ jets $)$ \\
$E_{T}^{\text {miss }} / m_{\text {eff }}^{\text {Njet }}>$ & $0.3 / 0.4 / 0.4$ & $0.25 / 0.3 /-$ & $0.25 / 0.3 / 0.3$ & 0.15 & $0.15 / 0.25 / 0.3$ \\
$m_{\text {eff }}^{\text {incl. }}[\mathrm{GeV}]>$ & $1900 / 1300 / 1000$ & $1900 / 1300 /-$ & $1900 / 1300 / 1000$ & $1700 /-/-$ & $1400 / 1300 / 1000$ \\
\hline Backgr. (tight) & $14 \pm 5$ & $8.7 \pm 3.4$ & $2.8 \pm 1.2$ & $6.3 \pm 2.1$ & $10 \pm 4$ \\
Data (tight) & 10 & 7 & 1 & 5 & 9 \\
\hline
\end{tabular}

Table 2: Summary of the signal regions categorised by numbers of jets and tight/medium/loose cuts for the search for quarks and gluinos with jets and $E_{\mathrm{T}}^{\text {miss }}$.

signal and control regions are performed to normalise the backgrounds directly from data. The cuts are optimised for $8 \mathrm{TeV}$ centre-of-mass energy. The $\Delta \phi$ cut to remove jets that are aligned with the $E_{\mathrm{T}}^{\text {miss }}$ direction and the $E_{\mathrm{T}}^{\text {miss }} / m_{\text {eff }}$ cut are effective in rejecting multijet background. The tight cuts give the maximum reach for high mass squarks and gluinos, while the medium and loose cuts add sensitivity for compressed spectra. No significant excess of events is observed in any channel. Example control and signal region distributions for the tight cuts are shown in Fig. 15. The resulting exclusions in the squark-gluino mass plane of the simplified model are shown in Fig. 16. The limits are stable up to an LSP mass of about $200 \mathrm{GeV}$.
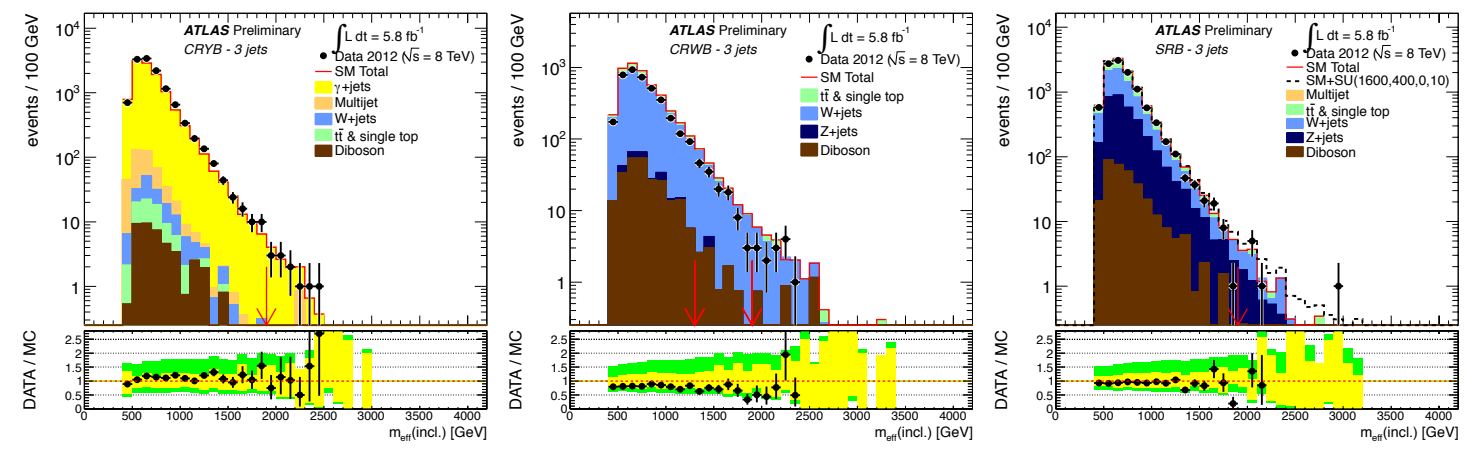

Figure 15: Two example control regions with tight selection cuts: $\gamma+$ jets to control the modelling of $Z \rightarrow v v+$ jets (left) and $\ell+E_{\mathrm{T}}^{\text {miss }}+$ jets with no $b$-tag to control $W+$ jets (centre). The corresponding signal region is shown on the right.

This search is complemented by a multijet analysis [20], optimised for long decay chains and requiring six or more jets. The ratio $E_{\mathrm{T}}^{\mathrm{miss}} / \sqrt{H_{\mathrm{T}}}$ is examined, where $H_{\mathrm{T}}=\sum_{i}\left|\vec{p}_{\mathrm{T}}{ }^{\text {jet }, i}\right|$. This gives a measure of the significance of the $E_{\mathrm{T}}^{\text {miss }}$ in the event. The distribution of the number of jets in events with large $E_{\mathrm{T}}^{\text {miss }} / \sqrt{H_{\mathrm{T}}}$ is shown in Fig. 17, together with the excluded region in a simplified model. The limit on the gluino mass is above $1 \mathrm{TeV}$ for an LSP mass below $300 \mathrm{GeV}$. 


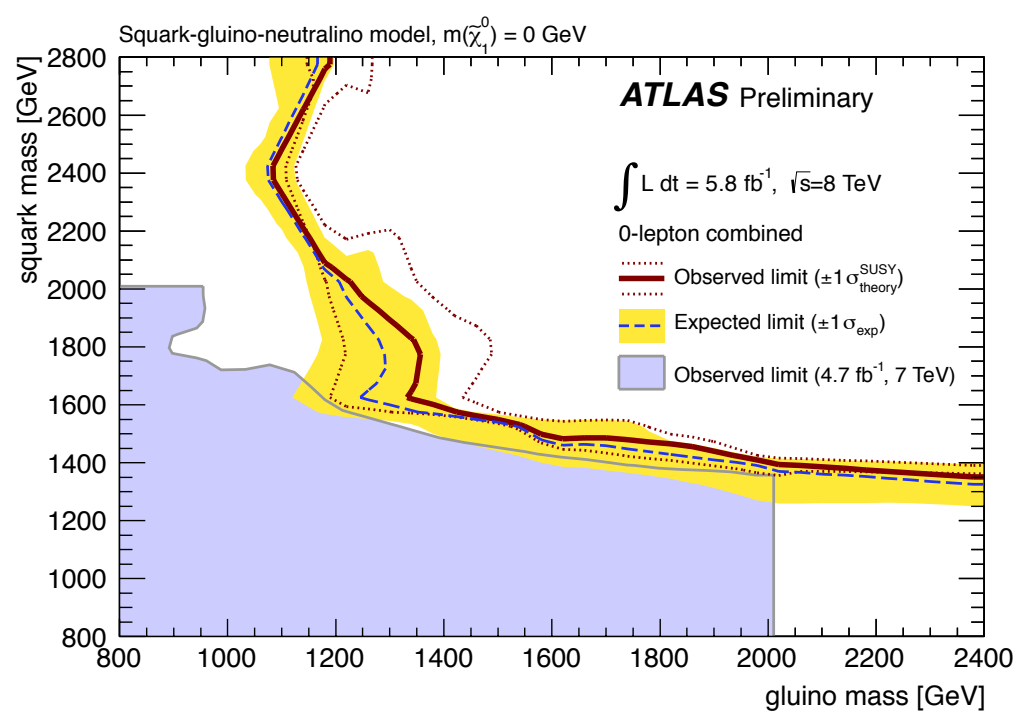

Figure 16: Excluded region in the squark-gluino mass plane for the simplified model, from the jets plus $E_{\mathrm{T}}^{\mathrm{miss}}$ analysis. The region excluded by the $7 \mathrm{TeV}$ data is also shown for comparison.
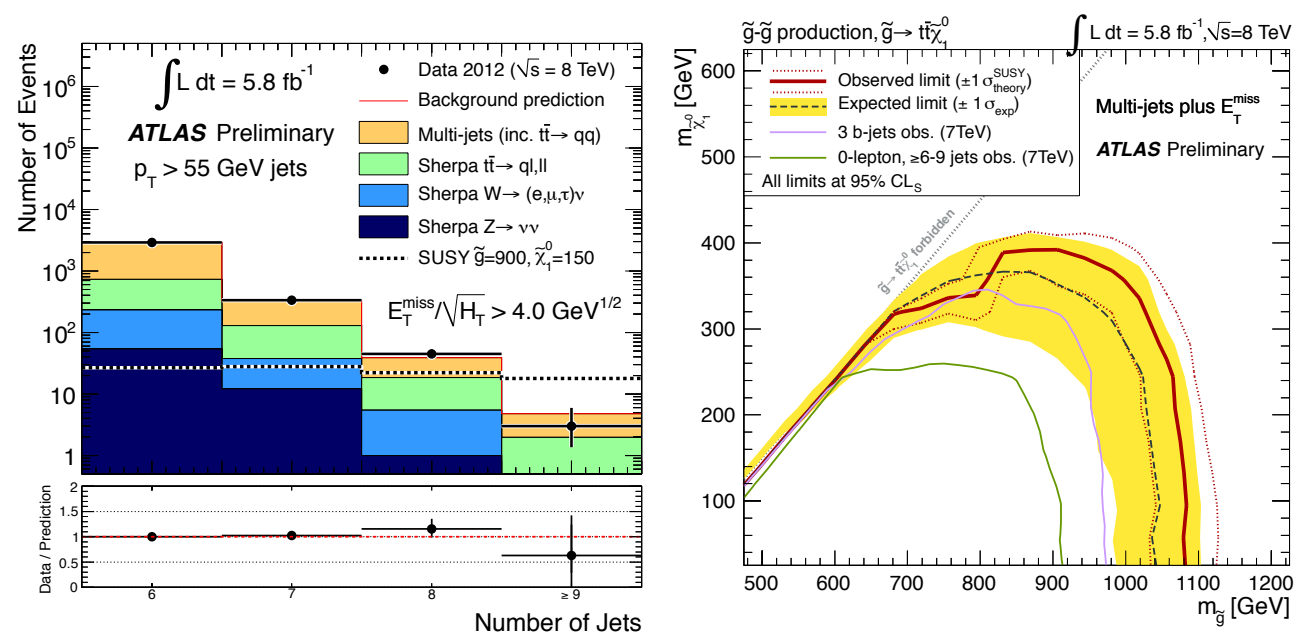

Figure 17: The distribution of the number of jets in events with significant $E_{\mathrm{T}}^{\mathrm{miss}}$, defined by a cut on $E_{\mathrm{T}}^{\text {miss }} / \sqrt{H_{\mathrm{T}}}>4 \sqrt{\mathrm{GeV}}$ (left), and the excluded region as a function of LSP and gluino mass in a simplified model (right) from the SUSY multijet search.

SUSY decay chains may also result in a lepton and $E_{\mathrm{T}}^{\text {miss }}$ in the final state from the decay of a chargino or slepton, for example $\tilde{q} \rightarrow q \tilde{\chi}_{1}^{-}$with $\tilde{\chi}_{1}^{-} \rightarrow \ell^{-} v \tilde{\chi}_{1}^{0}$. In this case the events can be selected with a lepton trigger, and the reduced multijet background allows more relaxed cuts to be applied. In the CMSSM interpretation, the 1-lepton search is competitive with the 0-lepton search for high $m_{0}$ [21]. A cascade decay including charginos and sleptons can result in dilepton final states. These may also arise directly from weak pair-production. The different configurations of same and opposite-sign, same and "opposite" $(e$ vs. $\mu)$ flavour, are exploited to exclude or enhance 
different processes. Results using $8 \mathrm{TeV}$ data and same-sign leptons accompanied by jets and $E_{\mathrm{T}}^{\text {miss }}$ are used to put limits on gluino decays via a top squark into $t \bar{t} \tilde{\chi}_{1}^{0}[22]$.

A novel alternative approach is to perform a generalised search [23]. The data are divided by event topology, according to the number of electrons, photons, muons, jets, $b$-jets and the value of $E_{\mathrm{T}}^{\text {miss }}$, resulting in 655 exclusive channels. An effective mass $m_{\mathrm{eff}}$ distribution is formed in each category, and compared with the distribution expected from Standard Model processes. No excess of events is found, and limits can be placed on particular SUSY models. The constraints are less sensitive for a specific model than those from dedicated searches, but this approach is more comprehensive.

Mixing in the third generation can lead to light SUSY partners, $\tilde{t}_{1}, \tilde{b}_{1}$ or $\tilde{\tau}_{1}$. Such models are appealing, in that they can lead to similar top and stop masses, favoured by naturalness arguments. The stop and sbottom may be prodcued in pairs, or via gluino decay. Searches for third generation squarks take advantage of the presence of $b$-jets in the final state, possibly from top-quark decay. The interpretation depends on the mass hierarchy and the decay modes. The results for stop pair production are summarised in Fig. 18 [24, 25, 26, 27, 28]. These are interpretations of searches with 0,1 or 2 leptons and $b$-jets in the final state.

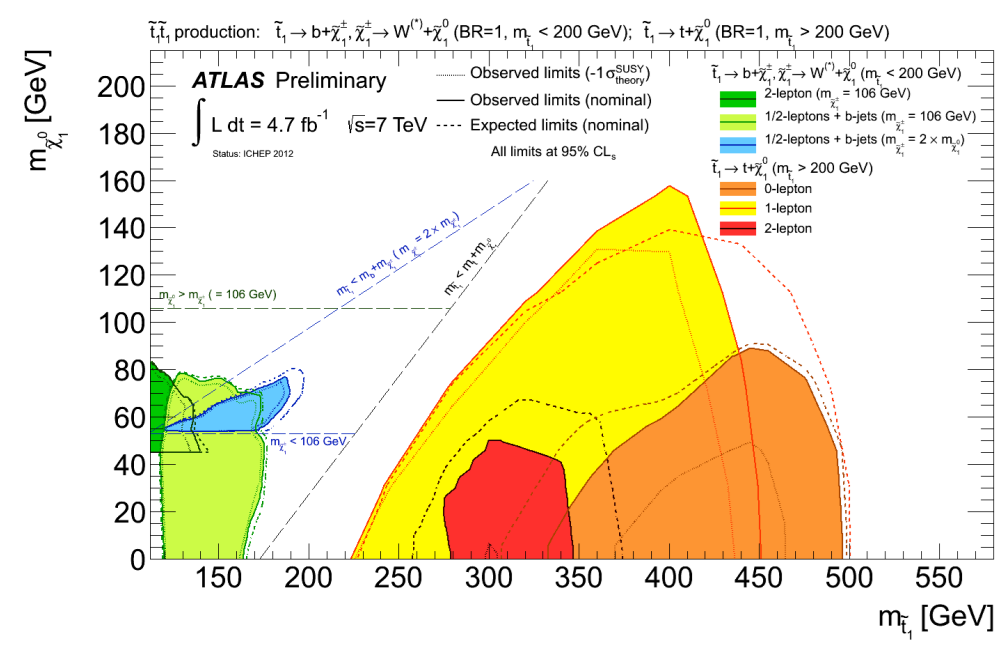

Figure 18: Summary of exclusion limits for stop-pair production as a function of the LSP and stop masses. The exclusions depend on the mass hierarchy. On the left side of the plot are the limits if $m(\tilde{t})<200 \mathrm{GeV}$, either assuming $m\left(\tilde{\chi}^{ \pm}\right)=106 \mathrm{GeV}$, or $m\left(\tilde{\chi}^{ \pm}\right)=2 m\left(\tilde{\chi}^{0}\right)$, with the decay $\tilde{t} \rightarrow b \tilde{\chi}^{ \pm}, \tilde{\chi}^{ \pm} \rightarrow W \tilde{\chi}^{0}$. On the right hand side, the stop is heavier, $m(\tilde{t})>200 \mathrm{GeV}$, and $\tilde{t} \rightarrow t \tilde{\chi}^{0}$.

In summary, despite a wide ranging search for hints of supersymmetry, no signals have emerged from the data analysed so far. In Fig. 19, the number of searches and interpretations is indicated with a typical exclusion limit for each [2]. Dedicated searches, for example for long-lived particles are included.

Further searches have been performed for many "exotic" phenomena, this being a generic term used to group non-SUSY and non-Higgs searches. These can be characterised by searches for new resonances, or "bump-hunting", in mass distributions including jets, leptons, photons and $t \bar{t}$. Further sensitivity to new phenomena comes from looking for deviations from the Standard Model 
expectation in angular distributions of final state objects. In addition, spectacular signatures, for example from the decay of mini-black holes can be sought. In general, the topologies of Higgs and SUSY final states can also be relevant for other models.

For example, the high mass end of the diphoton mass spectrum [29] can be used to search for extra dimensions, where the Arkani-Hamed, Dimopoulos and Dvali model predicts closely spaced resonances which look like a continuum distribution with an effective cut off scale, $M_{s}$. Limits on $M_{s}$ in the range $2.62-3.92 \mathrm{TeV}$ have been derived. Randall-Sundrum gravitons would be manifest as narrower resonances, a few $\mathrm{TeV}$ apart. Limits on the mass of the lightest graviton have been set of 1.00 (2.06) $\mathrm{TeV}$ for values of the coupling parameter $k / M_{\mathrm{Pl}} 0.01(0.1)$.

A search for high-mass $t \bar{t}$ resonances using final states with a lepton+jets has also been performed. Boosted top quarks can be reconstructed as fat jets. Kaluza-Klein gluons are excluded with a mass below $1.5 \mathrm{TeV}$ [30].

A summary of searches for exotic phenomena is given in Fig. 20. Limits range up to $10 \mathrm{TeV}$ in the case of contact interactions, which can have an influence below the centre-of-mass energy.

\section{Conclusions and outlook}

The LHC machine and ATLAS experiment have performed fantastically well at 7 and $8 \mathrm{TeV}$ centre-of-mass energy. The Standard Model stands firm, with good agreement between almost all measurements and the predictions. Further improvements in the description are expected with the increasing sophistication of calculations, including new Monte Carlo tunes and improved fits for parton distribution functions.

A new boson has been observed, with a mass of $126 \mathrm{GeV}$ and properties so far consistent with the Standard Model Higgs boson. Although no discoveries have been made so far in direct searches for SUSY particles or ohter physics beyond the Standard Model, the LHC has a long future, including a near doubling of the centre-of-mass energy, and much larger integrated luminosities. We are looking forward to the challenges and excitement of the coming years.

\section{Acknowledgements}

I would like to think my friends and colleagues in the ATLAS Collboration and in the CERN accelerator divisions for the spectacular performance and beautiful results from the last few years of LHC operation.

It is also a pleasure to thank the organisers for inviting me to the Corfu Summer Institute, and for their great Greek hospitality. 


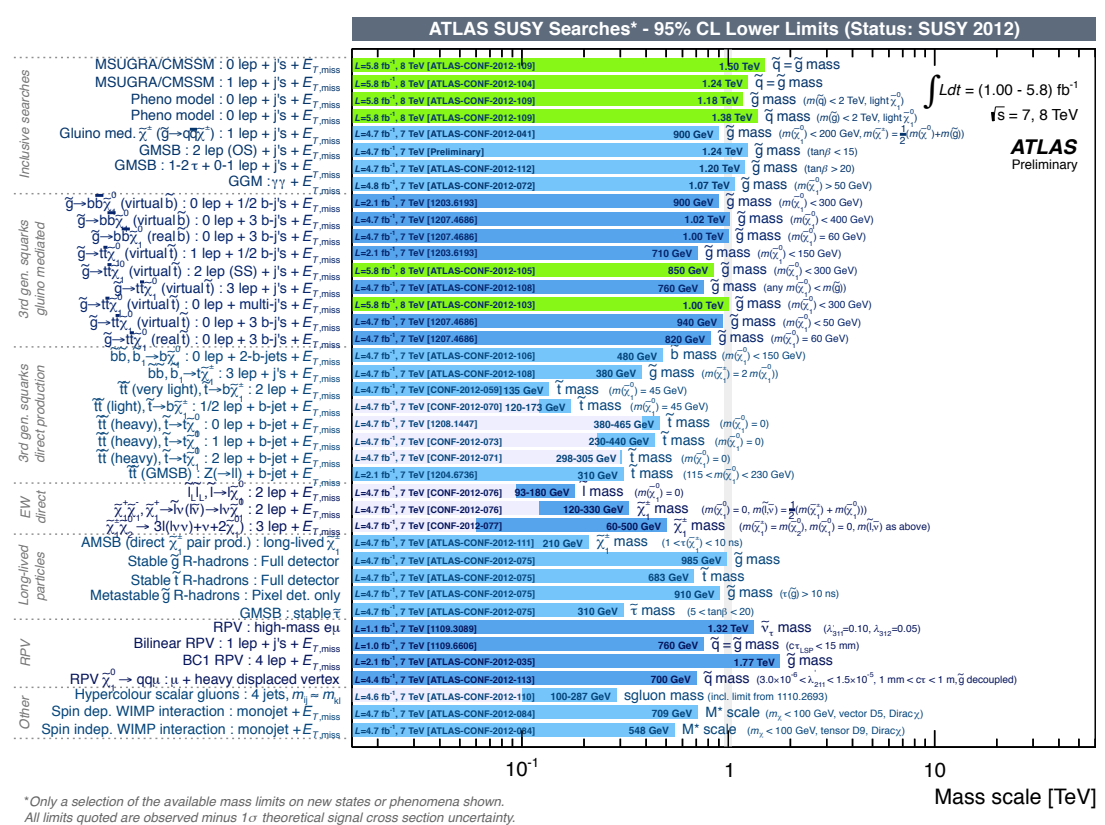

Figure 19: A summary of exclusion limits for SUSY signals. Strongly produced SUSY particles are typically excluded up to about $1 \mathrm{TeV}$ with the present data. The limits are lower for direct production of third generation squarks, or weakly produced particles.

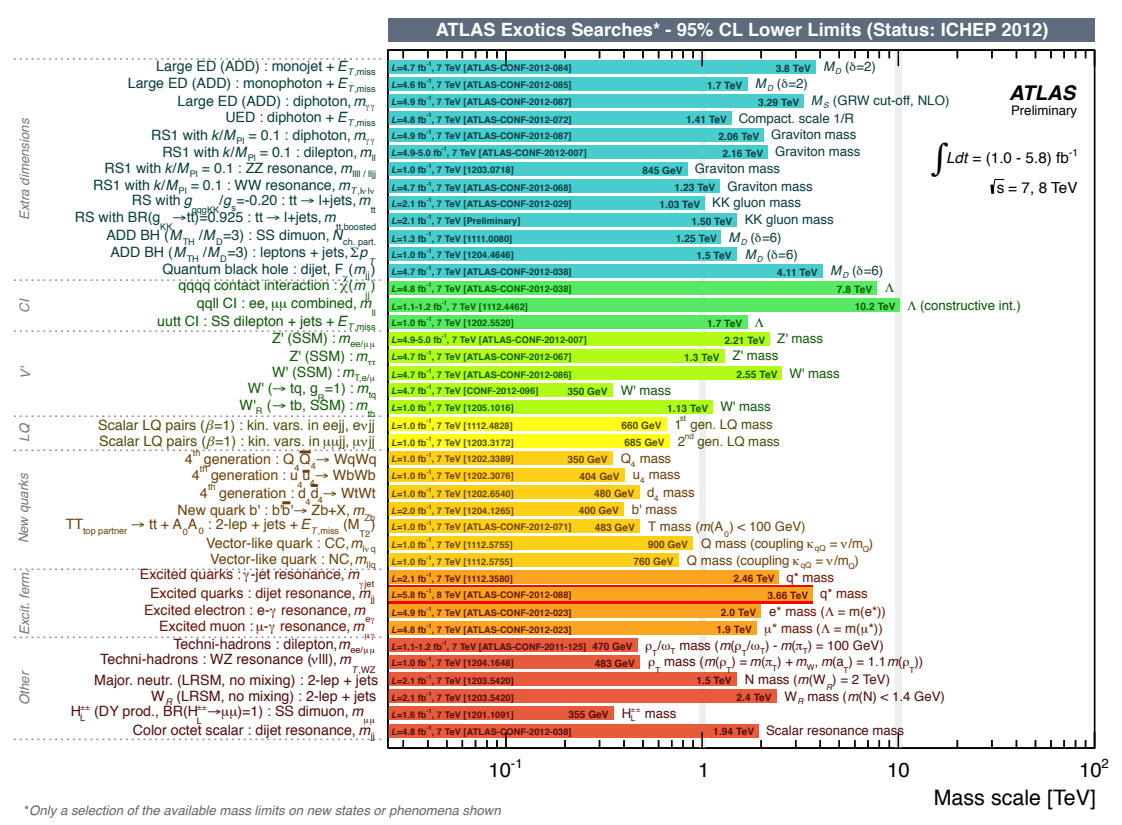

Figure 20: A summary of constraints from searches for exotic phenomena. 


\section{References}

[1] ATLAS Collaboration, The ATLAS Experiment at the CERN Large Hadron Collider, 2008 JINST 3 S08003.

[2] The results described in these proceedings are those that were available and presented at the time of the Corfu Summer Institute in September 2012. Links to all public results from the ATLAS Collaboration can be found here https://twiki.cern.ch/twiki/bin/view/AtlasPublic.

[3] ATLAS Collaboration, Charged-particle multiplicities in $p p$ interactions measured with the ATLAS detector at the LHC New J. Phys. 13 (2011) 053033.

[4] ATLAS Collaboration, Measurements of underlying-event properties using neutral and charged particles in $p p$ collisions at $900 \mathrm{GeV}$ and $7 \mathrm{TeV}$ with the ATLAS detector at the LHC, Eur. Phys. J. C71 (2011) 1636.

[5] ATLAS Collaboration, Measurement of inclusive jet and dijet production in $p p$ collisions at $\sqrt{s}=7 \mathrm{TeV}$ using the ATLAS detector, Phys. Rev. D86 (2012) 014022.

[6] ATLAS Collaboration, Measurement of multi-jet cross sections in proton-proton collisions at a $7 \mathrm{TeV}$ center-of-mass energy, Eur. Phys. J. C 71 (2011) 1763.

[7] ATLAS Collaboration, Search for New Phenomena in the Dijet Mass Distribution using $5.8 \mathrm{fb}^{-1} \mathrm{of}^{\circ}$ pp Collisions at $\sqrt{s}=8 \mathrm{TeV}$ collected by the ATLAS Detector, ATLAS-CONF-2012-088.

[8] ATLAS Collaboration, Search for New Phenomena in Dijet Mass and Angular Distributions using $4.8 \mathrm{fb}^{-1}$ of $p p$ Collisions at $\sqrt{s}=7 \mathrm{TeV}$ collected by the ATLAS Detector, ATLAS-CONF-2012-038.

[9] ATLAS Collaboration, A measurement of the inclusive $W^{ \pm}$and $Z / \gamma^{*}$ cross sections in the $e$ and $\mu$ decay channels in $p p$ collisions at $\sqrt{s}=7 \mathrm{TeV}$ with the ATLAS detector, Phys. Rev. D85 (2012) 072004.

[10] ATLAS Collaboration, Measurement of the transverse momentum distribution of $W$ bosons in $p p$ collisions at $\sqrt{s}=7 \mathrm{TeV}$ with the ATLAS detector, Phys. Rev. D 85 (2012) 012005.

[11] ATLAS Collaboration, Measurement of the production cross section for $W$-bosons in association with jets in $p p$ collisions using $33 \mathrm{pb}^{-1}$ of data at $\sqrt{s}=7 \mathrm{TeV}$ with the ATLAS detector, ATLAS-CONF-2011-060.

[12] ATLAS Collaboration, Measurement of $W Z$ production in proton-proton collisions at $\sqrt{s}=7 \mathrm{TeV}$ with the ATLAS detector, Eur. Phys. J. C 72 (2012) 2173.

[13] ATLAS Collaboration, Statistical combination of top quark pair production cross-section measurements using dilepton, single-lepton, and all-hadronic final states at $\sqrt{s}=7 \mathrm{TeV}$ with the ATLAS detector, ATLAS-CONF-2012-024.

[14] ATLAS Collaboration, Measurements of top quark pair relative differential cross-sections with ATLAS in $p p$ collisions at $\sqrt{s}=7$ TeV, Eur. Phys. J. C 73 (2013) 2261.

[15] ATLAS Collaboration, Measurement of the top quark mass with the template method in the $t \bar{t} \rightarrow \ell+$ jets channel using ATLAS data, Eur. Phys. J. C 72 (2012) 2046.

[16] ATLAS Collaboration, Measurement of the charge asymmetry in top quark pair production in $p p$ collisions at $\sqrt{s}=7 \mathrm{TeV}$ using the ATLAS detector, Eur. Phys. J. C 72 (2012) 2039

[17] LHC Higgs Cross Section Working Group, https://twiki.cern.ch/twiki/bin/view/LHCPhysics/CrossSections. 
[18] ATLAS Collaboration, Observation of a new particle in the search for the Standard Model Higgs boson with the ATLAS detector at the LHC, Phys. Lett. B 716 (2012) 1.

[19] ATLAS Collaboration, Search for squarks and gluinos with the ATLAS detector using final states with jets and missing transverse momentum and $5.8 \mathrm{fb}^{-1}$ of $\sqrt{s}=8 \mathrm{TeV}$ proton-proton collision data, ATLAS-CONF-2012-109

[20] ATLAS Collaboration, Search for new phenomena using large jet multiplicities and missing transverse momentum with ATLAS in $5.8 \mathrm{fb}^{-1}$ of $\sqrt{s}=8 \mathrm{TeV}$ proton-proton collisions ATLAS-CONF-2012-103.

[21] ATLAS Collaboration, Search for supersymmetry in final states with jets, missing transverse momentum and one isolated lepton in $\sqrt{s}=7 \mathrm{TeV} p p$ collisions using $1 \mathrm{fb}^{-1}$ of ATLAS data Phys. Rev. D 85 (2012) 012006.

[22] ATLAS Collaboration, Search for Supersymmetry in final states with two same-sign leptons, jets and missing transverse momentum with the ATLAS detector in $p p$ collisions at $\sqrt{s}=8 \mathrm{TeV}$, ALTAS-CONF-2012-105.

[23] ATLAS Collaboration, A general search for new phenomena with the ATLAS detector in $p p$ collisions at $\sqrt{s}=7 \mathrm{TeV}$, ATLAS-CONF-2012-107.

[24] ATLAS Collaboration, Search for light scalar top quark pair production in final states with two leptons with the ATLAS detector in $\sqrt{s}=7 \mathrm{TeV}$ proton-proton collisions, ATLAS-CONF-2012-059.

[25] ATLAS Collaboration, Search for light scalar top pair production in final states with leptons and $b$-jets with the ATLAS detector in $\sqrt{s}=7 \mathrm{TeV}$ proton-proton collisions, ATLAS-CONF-2012-070.

[26] ATLAS Collaboration, Search for a heavy top partner in final states with two leptons with the ATLAS detector ATLAS-CONF-2012-071.

[27] ATLAS Collaboration, Search for direct top squark pair production in final states with one isolated lepton, jets, and missing transverse momentum in $\sqrt{s}=7 \mathrm{TeV} p p$ collisions using $4.7 \mathrm{fb}^{-1}$ of ATLAS data, ATLAS-CONF-2012-073.

[28] ATLAS Collaboration, Search for a supersymmetric partner to the top quark in final states with jets and missing transverse momentum at $\sqrt{s}=7 \mathrm{TeV}$ with the ATLAS detector, ATLAS-CONF-2012-074.

[29] ATLAS Collaboration, Search for Extra Dimensions in the Diphoton Channel using $4.9 \mathrm{fb}^{-1}$ of Proton-Proton Collisions recorded at $\sqrt{s}=7 \mathrm{TeV}$ with the ATLAS Detector, ATLAS-CONF-2012-087.

[30] ATLAS Collaboration, A search for $t \bar{t}$ resonances in lepton+jets events with highly boosted top quarks collected in $p p$ collisions at $\sqrt{s}=7 \mathrm{TeV}$ with the ATLAS detector, JHEP 1209 (2012) 041. 